\title{
Categorization versus distance: Hemispheric differences for processing spatial information
}

\author{
JOSEPH B. HELLIGE and CHIKASHI MICHIMATA \\ University of Southern California, Los Angeles, California
}

\begin{abstract}
It has been hypothesized that the brain computes two different kinds of spatial-relation representations: one used to assign a spatial relation to a category and the other used to specify metric distance with precision. The present visual half-field experiment offers support for this distinction by showing that the left and right cerebral hemispheres make more effective use of the categorization and metric distance representations, respectively. Furthermore, the inclusion of a bilateral stimulus presentation condition permits the computation of a reversed association that offers additional support for the distinction between two types of spatial-relation representation.
\end{abstract}

Kosslyn (1987) has hypothesized that the human brain computes two different kinds of spatial-relation representations. One type of representation is used to assign a spatial relation to a category, such as "outside of" or "above," whereas the other type of representation preserves location information using a metric coordinate system in which distances are specified effectively. One way to obtain converging information about the plausibility of the distinction between these two processing subsystems is to show that they have different neurological substrata. With this in mind, Kosslyn has suggested that the left cerebral hemisphere makes more effective use of the categorization processing subsystem and that the right cerebral hemisphere makes more effective use of the metric distance processing subsystem. The present experiment was designed to provide tests of this lateralization hypothesis.

On each trial of the present experiment, the subjects were shown a stimulus consisting of a horizontal line and a small dot in one of 12 possible locations ( 6 above the line and 6 below the line). During different experimental sessions, each subject performed a categorization task and a distance judgment task using these stimuli. Stimuli on different trials were presented to the right visual field-left hemisphere (RVF-LH) or the left visual field-right hemisphere (LVF-RH). It was of particular interest to determine whether or not there would be a task $\times$ visual field interaction (a processing dissociation, as discussed by Hellige, 1983, and Zaidel, 1983) and whether the interaction would be of the form predicted by Kosslyn's (1987) hypothesis. Because the stimuli and responses are identical for the categorization and distance-judgment tasks of the present experiment, any such interaction must

The research reported here was supported in part by Research Grant BNS-8608893 from the National Science Foundation. The authors thank Alice Healy, Stephen Kosslyn, and two anonymous reviewers for helpful comments on an earlier draft of this manuscript. Correspondence may be sent to Joseph B. Hellige, Department of Psychology, University of Southern California, Los Angeles, CA 90089-1061. reflect differences in the processing subsystems required rather than other differences related to stimulus input (cf. Hellige \& Sergent, 1986; Sergent \& Hellige, 1986).

During the categorization task, the subjects were required to indicate whether or not the dot on each trial was above or below the line, ignoring the distance between the dot and the line. This above/below task is conceptually similar to two categorization tasks (the on/off and left/right tasks) described by Kosslyn (1987), who also summarized experiments showing that those tasks produce a RVF-LH advantage in visual half-field studies with neurologically normal subjects.

During the distance-judgment tasks, the subjects were required to indicate whether or not the dot on each trial was within approximately $2 \mathrm{~cm}$ of the line on either side. To do this, the subjects were trained to refer to the six dot locations nearest the line on either side (all within $2 \mathrm{~cm}$ of the line) as "near" and to refer to the six dot locations farthest from the line on either side (all farther than $2 \mathrm{~cm}$ from the line) as "far." This near/far task is similar to the two distance-judgment tasks described by Kosslyn (1987), both of which required that subjects indicate whether two stimuli were within a specified distance (either $2 \mathrm{~mm}$ or $1 \mathrm{in}$.) of each other. Thus, the near/far task meets Kosslyn's criterion for involving a distance judgment of the sort that has been hypothesized and reported to produce an LVF-RH advantage. However, use of the near/far labels ensures that any differences in lateralization for the category and distance tasks cannot be attributed to the use of readily available verbal labels in one case but not in the other.

It is instructive to note that, in the results summarized by Kosslyn (1987), the specific categorization tasks used were more difficult than were the specific distancejudgment tasks. This raises the possibility that the different visual-field advantages for the tasks were produced by different levels of difficulty rather than by the task demands that he emphasizes. With this in mind, the present tasks were chosen on the basis of pilot work, so that the above/below categorization task would be easier than 
would the near/far distance-judgment task. To the extent that the present experiment produces a task $x$ visual field interaction of the sort predicted by Kosslyn, it would be difficult to explain both his data and the present data on the basis of task difficulty.

The present experiment also differed from those summarized by Kosslyn (1987) by making the type of task a within-subjects variable. This was done to allow an investigation of individual differences among right-handers in the magnitude of hemispheric dominance for the two hypothesized aspects of spatial processing. In his 1987 article, Kosslyn suggested that hemispheric asymmetries arise as a function of different "seeding" of the two hemispheres early in development. On the basis of the notion of individual differences in the extent of seeding, Kosslyn suggested that individuals who show a relatively large RVF-LH advantage for categorization tasks should also show a relatively large LVF-RH advantage for distance-judgment tasks. He provided some evidence for this by summarizing a study showing larger laterality effects of both sorts for strongly right-handed subjects than for ambidextrous subjects, with the assumption being that different seeding of the two hemispheres is more likely in the strongly right-handed group.

Even within the right-handed population, the magnitude of hemispheric dominance for each task is assumed to reflect the extent of seeding differences for the two hemispheres. Furthermore, recent studies (e.g., Hellige, Bloch, \& Taylor, 1988; Levine, Banich, \& Kim, 1987; Levy, Heller, Banich, \& Burton, 1983a, 1983b) suggest considerable individual variation among right-handers in the direction and magnitude of cerebral laterality for a variety of verbal and nonverbal tasks. In view of this variability and in view of Kosslyn's (1987) results for right-handed versus ambidextrous subjects, the present experiment tests the hypothesis that right-handers who show a relatively large laterality effect for one task also show a relatively large laterality effect in the opposite direction for the other task.

In addition to including LVF-RH and RVF-LH stimulus presentations, the present experiment also included trials on which the identical stimulus was presented simultaneously to both visual fields (bilateral trials). This was done for the following reasons. As discussed in more detail later, the inclusion of bilateral trials allows a stronger test of the distinction between two kinds of spatial processing by taking advantage of the reversed association logic developed by Dunn and Kirsner (1988). In addition, the presence of a task $\times$ visual field interaction on unilateral trials would suggest qualitatively different processing propensities in the left and right cerebral hemispheres. By comparing the pattern of results on bilateral trials with the pattern on LVF-RH and RVF-LH trials, it is possible to examine whether subjects are equally influenced by the LVF and RVF stimuli in a condition for which the same information is presented simultaneously to both fields. To the extent that the pattern of results on bilateral trials matches that of one unilateral visual field but not the other, we have some evidence about which hemisphere exerts control over the task under conditions that allow either hemisphere to do so (see Hellige, 1987; Hellige, Jonsson, \& Michimata, 1988; Hellige \& Michimata, 1989; Hellige, Taylor, \& Eng, in press).

\section{METHOD}

\section{Subjects}

Forty-six volunteers from introductory psychology classes $(22$ men, 24 women) with normal or corrected-to-normal visual acuity participated in the experiment. All subjects were right-handed as assessed by the Edinburgh Handedness Inventory (Oldfield, 1971). Preliminary analyses of variance indicated no main effects or interactions related to subjects' gender, so the results for men and women have been combined

\section{Apparatus}

The subject sat at a table facing a $44 \times 48 \mathrm{~cm}$ screen approximately $60 \mathrm{~cm}$ away. A black posterboard covered the screen, with two rectangular windows cut out so that stimuli could be presented to the left and right visual fields. Each window measured approximately $12 \mathrm{~cm}$ vertically $\times 4 \mathrm{~cm}$ horizontally. The edge of each window nearest the center of the screen was approximately $1.7 \mathrm{~cm}$ from the center. Midway between these two windows was a small circular opening for projection of a fixation dot. During the experiment, the subject's chin was placed on a padded rest with a foreheadstabilization bar in order to ensure that the subject's midline was perpendicular to the viewing screen. Centered on the table in front of the subject was a $17 \times 35.5 \mathrm{~cm}$ response console. On top of the console were four buttons arranged in a row from left to right. The buttons were arranged into two pairs with the centermost button of each pair $7.5 \mathrm{~cm}$ from the center of the console. The two buttons within a pair were $7.5 \mathrm{~cm}$ apart. For the above/below task, a small card with the same label (ABOVE or BELOW, counterbalanced across subjects) was placed above the two innermost buttons and a small card with the opposite label was placed above the two outermost buttons. For the near/far task, the arrangement was similar, but with the two labels being NEAR and FAR. Visual stimuli and a fixation dot were rear-projected onto the viewing screen at the appropriate times using a Gerbrands three-field tachistoscope (Model G1176) equipped with two Kodak Carousel 850 slide projectors with Kodak Ektanar $\mathrm{f} / 2.8$-in. lenses. Stimulus duration was controlled by a Gerbrands six-channel timer (Model 300-6T). Summary statistics for each experimental session were computed by an Apple Ile microprocessor.

\section{Stimulus Materials}

The stimulus on each trial consisted of a horizontal line positioned even with the fixation dot (i.e., midway between the top and bottom edge of the viewing window) and a small dot located in one of 12 possible positions above and below the line (see Figure 1). The dot subtended approximately $0.2^{\circ}$ of visual angle. Beginning with the position nearest the line, the possible positions of the dot were approximately $0.5^{\circ}, 1.0^{\circ}, 1.5^{\circ}, 2.5^{\circ}, 3.0^{\circ}$, and $3.5^{\circ}$ of visual angle from the line. When projected onto the screen, the line and dot appeared as white stimuli with a luminance of approximately $4.0 \mathrm{~cd} / \mathrm{m}^{2}$ on an opaque background. On different trials, the stimulus was projected to only the LVF-RH window, to only the RVF-LH window, or to both windows simultaneously (bilateral trials). Note that, on bilateral trials, the same stimulus was presented to both windows. The centermost edge of each of these lateralized stimuli was approximately $2.0^{\circ}$ of visual angle from the center of the screen. A fixation dot subtending approximately $0.2^{\circ}$ of visual angle with a luminance of approximately $4.0 \mathrm{~cd} / \mathrm{m}^{2}$ was projected at appropriate times to the center of the viewing screen. 


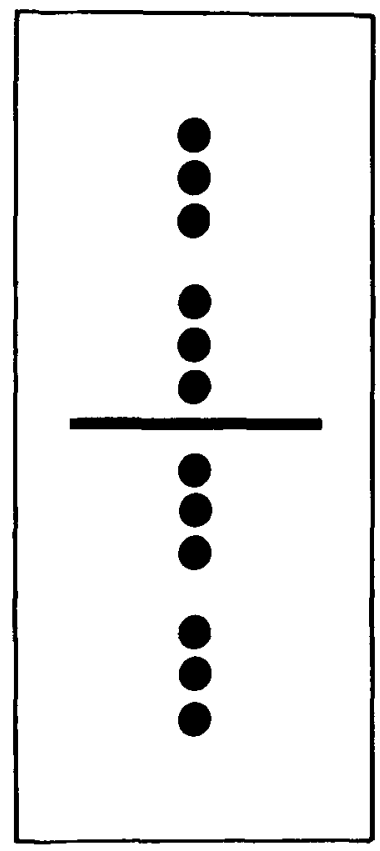

Figure 1. Illustration of the $\mathbf{1 2}$ possible dot locations used in the present experiment.

During each of the two experimental tasks, the subjects received a total of 144 experimental trials consisting of a random ordering of four 36-trial sets. Each of these sets consisted of each of the 12 possible dot positions presented one time in each of the three visualfield conditions. Within a 36-trial set, the trial types were arranged randomly, with the restriction that no visual-field condition occur more than four times in a row.

\section{Procedure}

At the beginning of each experimental session, the subjects were told to keep the index and middle fingers of the left and right hands on the innermost and outermost response keys, respectively, and to direct their gazes toward the fixation dot when it appeared. The subjects were told to maintain that eye fixation until after they had made their responses on each trial. In addition, the subjects were told that the stimulus item would sometimes appear in the left window only, sometimes appear in the right window only, and sometimes appear in both windows at the same time. It was stressed that when both windows contained information, it would always be the case that the same stimulus was present in both windows. While they were being instructed about the experimental task, each subject was given a sheet containing all 12 possible stimuli divided into the four categories defined by the orthogonal combination of whether the dot was above or below the line and whether the dot was near the line or far from the line.

For the above/below task, the subjects were told to indicate as quickly and as accurately as possible whether the dot was above or below the line. The subjects were told that the distance between the dot and the line would vary, but that distance was irrelevant to the task. The subjects made their responses by simultaneously pressing both index fingers or both middle fingers, with the assignment of fingers to above/below responses counterbalanced across subjects. This response procedure was used because it allows each hemisphere to have access to both responses on each trial (see Hellige \& Sergent, 1986). This may be particularly important when using bilateral trials to examine whether or not one hemisphere's preferred mode of processing dominates when both hemispheres have access to the same information (e.g., Hellige \& Michimata, 1989). In the event that the two buttons were not pressed with perfect simultaneity, reaction time (RT) was defined by the time of the first press. Each trial began with the onset of the fixation dot for $1 \mathrm{sec}$ followed immediately by the stimulus item for $150 \mathrm{msec}$. The intertrial interval was $5 \mathrm{sec}$. Prior to the experimental trials, the subjects received a set of 36 practice trials to be certain that they understood the task and to become familiar with the response procedures.

During a second experimental session, approximately $48 \mathrm{~h}$ after the first, the subjects were given the near/far task. For this task, the subjects were taught to consider the three dot locations nearest the line on either side (i.e., within $2 \mathrm{~cm}$ ) to be near the line and to consider the three dot locations farthest from the line on either side to be far from the line, with the above/below dimension being irrelevant. The subjects were instructed to press either the two buttons labeled NEAR (both index or middle fingers, counterbalanced across subjects) or the two buttons labeled FAR as quickly and as accurately as possible to make their responses on each trial. Other aspects of the procedure were identical to those of the above/below task, with one exception. During the 36 practice trials for the near/far task, the subjects were given feedback after every trial about the correctness of their responses in an effort to teach them the critical distance that separated "near" from "far."

Ordinarily, one might counterbalance the order of the above/below and near/far tasks. This was not done for the following reason. One purpose of this experiment was to examine whether or not individuals who show a relatively large RVF-LH advantage for the above/below task would show a relatively large LVF-RH advantage for the near/far task. When investigating such individual differences, it is desirable to order tasks in the same way for all subjects so as to hold any order and carryover effects constant (cf. Hellige, Bloch, \& Taylor, 1988). To minimize such effects, the testing sessions were separated by $48 \mathrm{~h}$. Note that the task performed second might benefit from practice with the task performed first or might suffer from fatigue or negative transfer for those stimuli assigned to different response keys in the two tasks. Given the 48-h separation between the tasks and the fact that the above/below and near/far responses were orthogonal and conceptually distinct, it seemed more likely that performance during the second session would, if anything, benefit from practice with the stimuli more than suffer from fatigue or negative transfer. This being the case, the above/below task was administered first because pilot work had indicated that it was the easier of the two tasks, rarely leading to an error and producing relatively short RTs. Thus, any advantage for the above/below task in the present experiment would have to occur despite the fact that the near/far task could benefit from being administered second.

\section{RESULTS AND DISCUSSION}

For each subject during each of the two tasks, the percentage of errors and the median RT of correct responses were computed for each visual-field condition (LVF-RH, RVF-LH, and bilateral). There was no evidence for a different speed/accuracy tradeoff for the two tasks or for the three visual-field conditions. In fact, the pattern of results was very similar for percentage of errors and RTs, producing a correlation of $r=+.974$ between the mean $\mathrm{RT}$ and the mean percentage of errors for the six trial types defined by the orthogonal combination of two tasks and three visual-field conditions. The percentage of errors was too low for the above/below task $(M=2.87 \% ; S D=4.31)$ to allow a meaningful analysis of either visual-field differences in the error rate for that task or a comparison of the error rate pattern for the two tasks. The higher percentage of errors for the near/far task $(M=9.17 \%$; 
$S D=6.52$ ) does allow for a meaningful analysis of visualfield differences in the error rate for that task. However, in view of the generally low error rates and the floor effect for errors for the above/below task, emphasis is placed on RT as the primary dependent variable.

Figure 2 shows the mean of median RTs for each of the three visual-field conditions in each of the two tasks. The RT values were subjected to an analysis of variance with task (above/below vs. near/far) and visual-field condition (LVF-RH, RVF-LH, and bilateral) as repeated measures variables. As Figure 2 shows, responses were faster for the above/below task than for the near/far task $\left[F(1,45)=23.14, M S_{\mathrm{e}}=28,255.5, p<.001\right]$. This finding is consistent with the lower error rate for the above/below task, and both results indicate that, in the present experiment, the categorization task was easier than was the distance-judgment task. This effect occurred despite the fact that the near/far task was always administered during the second session when the subjects were more familiar with the stimuli and response procedure; this confirms a similar observation about task difficulty made in the pilot work that led to the specific task parameters that were used. There was also a significant main effect of visual field $\left[F(2,90)=12.49, M S_{\mathrm{e}}=923.0\right.$, $p<.001]$, with RTs being faster for bilateral presentations $(M=614 \mathrm{msec})$ than for LVF-RH $(M=632 \mathrm{msec})$ or RVF-LH $(M=636 \mathrm{msec})$ presentations, with the latter two conditions not differing. The beneficial effect of having two completely redundant stimuli (bilateral trials) is not surprising, but it has not always been found (e.g., Hellige, Jonsson, \& Michimata, 1988). Of particular theoretical importance was a significant task $\times$ visual field interaction $\left[F(2,90)=5.04, M S_{\mathrm{e}}=708.2, p<.01\right]$. To clarify the nature of this interaction, LVF-RH versus

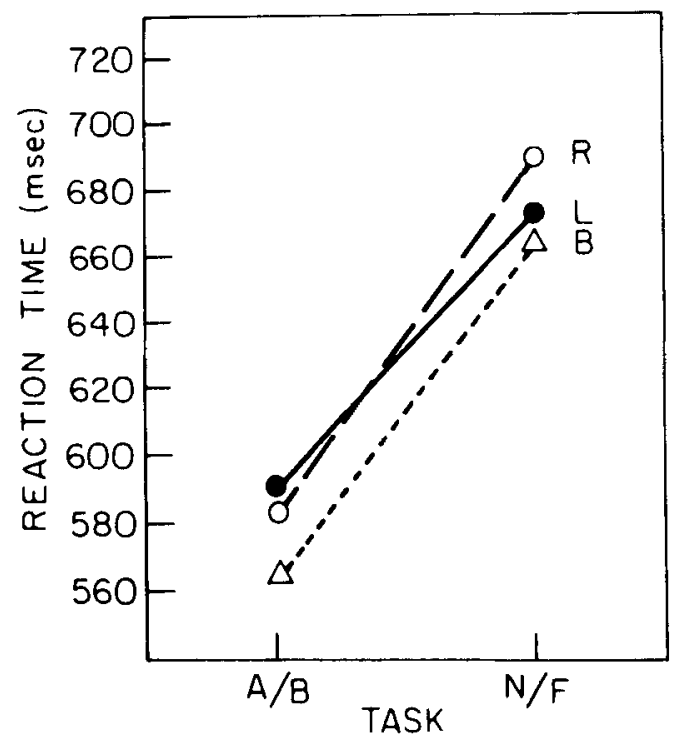

Figure 2. Reaction time for the above/below (A/B) and near/far (N/F) tasks. The parameter is visual-field condition: RVF-LH (R), LVF-RH (L), and bilateral (B).
RVF-LH trials will be considered first followed by a consideration of bilateral trials.

When analysis is restricted to the two unilateral visual fields, the task $\times$ visual field interaction continues to be significant $\left[F(1,45)=8.74, M S_{\mathrm{e}}=639.2, p<.005\right]$. The form of this interaction is consistent with the hypothesis of left-hemisphere dominance for the above/below categorization task and right-hemisphere dominance for the near/far distance-judgment task. That is, there was a marginally significant RVF-LH advantage for the above/below task $[t(45)=1.48, S E=4.56, p<.10$, one-tailed] and a significant LVF-RH advantage for the near/far task $[t(45)=2.30, S E=6.41, p<.015$, onetailed]. The LVF-RH advantage for the near/far task is corroborated by an analysis of the percentage of errors for that task, with there being significantly fewer errors on LVF-RH trials $(8.15 \%)$ than on RVF-LH trials $(11.25 \%)\left[F(1,45)=36.29, M S_{\mathrm{e}}=24.7, p<.001\right]$.

As argued in the introduction, a processing dissociation of the sort found in the task $\times$ visual field interaction for unilateral trials is suggested by Kosslyn (1987) and others to indicate that the two tasks are handled by qualitatively different information-processing mechanisms. In a thought-provoking logical analysis, Dunn and Kirsner (1988) argue that such processing dissociations do not unequivocally rule out the interpretation that two tasks are mediated by a common mechanism. A complete review of their logic is beyond the scope of this article, but one important notion is that two tasks may be of such different levels of difficulty that they produce different patterns of effects (such as, visual-field effects) despite being mediated by the same information-processing component. From this point of view, a unilateral visual field $x$ task interaction does not provide unequivocal evidence that the two spatial tasks are mediated by different processing mechanisms.

While they caution against the overuse of processing dissociations, Dunn and Kirsner (1988) argue that another criterion is sufficient to provide unequivocal evidence that two tasks are mediated by qualitatively different mechanisms. They refer to that criterion as reversed association. To determine whether a reversed association exists, it is necessary to covary the two tasks of interest with some other independent variable that has at least three conditions. This independent variable can be either quantitative (e.g., see Dunn and Kirsner's, 1988, example in their Figure 4) or categorical (e.g., see Dunn and Kirsner's example in their Figure 6 using various amnesic and control groups and related vs. unrelated word lists). The inclusion of bilateral as well as two unilateral trial types allows visual-field condition to be used in this way for the present experiment. In looking for a reversed association, performance on one task is plotted as a function of performance on the other task. Such a plot is shown for the present experiment in Figure 3, which shows RT for the above/below task on the horizontal axis and RT for the near/far task on the vertical axis. Each of the three data 


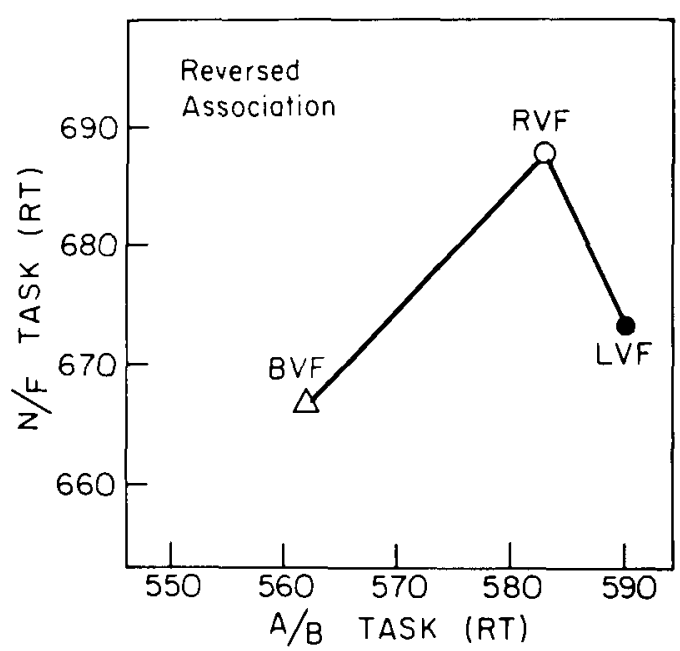

Figure 3. Reaction time for the near/far task (N/F) plotted as a function of reaction time for the above/below task (A/B). The data points show the reaction times for each task during each of the three visual-field conditions: RVF-LH (RVF), LVF-RH (LVF), and bilateral (BVF).

points comes from one of the three visual-field conditions and represents the RTs for the two tasks in that visualfield condition. As Dunn and Kirsner argue, if the two tasks are mediated by a single processing mechanism, data plotted in this way must be monotonic. Accordingly, deviations from monotonicity provide unequivocal evidence for two qualitatively different processing mechanisms.

When viewed in this way, the data in Figure 3 tell an interesting story. Note that, for the above/below task, mean RTs were significantly different for the three visualfield conditions $\left[F(2,90)=13.94, M S_{e}=732.1\right]$, with the mean RT being faster on bilateral trials than on either RVF-LH or LVF-RH trials and faster on RVF-LH trials than on LVF-RH trials. Now consider the RT results for the near/far task, moving left to right across the above/below axis. The mean near/far RT on bilateral trials was significantly less than the mean near/far RT on RVF-LH trials $[t(45)=4.38, S E=4.86, p<.01]$. In order for monotonicity to be preserved, the mean near/far RT on LVF-RH trials must be equal to or greater than the mean near/far RT on RVF-LH trials. However, we saw earlier that the mean near/far RT is significantly less on LVF-RH trials than on RVF-LH trials. Thus, we have clear evidence of a statistically significant deviation from monotonicity in Figure 3. The presence of such a reversed association offers strong converging evidence for a twoprocess hypothesis.

Because the visual-field effects are consistent with Kosslyn's (1987) two-process theory, it is possible to use these tasks to test the hypothesis that right-handers who show relatively large RVF-LH advantages for a categorization task also show relatively large LVF-RH advantages for a distance-judgment task. To test this prediction, the RVF-minus-LVF RT difference score was computed for each subject during each task and the two differences were correlated. According to the hypothesis just considered, there should be a significant negative correlation. Instead, there was a nonsignificant positive correlation of $r=+.159$.

The absence of the hypothesized negative correlation is inconsistent with a prediction made by Kosslyn (1987). However, a recent computer simulation of Kosslyn's theory (Kosslyn, Sokolov, \& Chen, in press) suggests that the prediction about individual differences may not follow from the 1987 theory. Instead, the simulation suggests that the theory actually predicts that the two kinds of laterality effects should be independent of each other. The fact that the correlation in the present experiment was not significantly different from zero could, therefore, be taken as support for the independence prediction.

However, the consistency of individual differences across laterality tasks merits further study for several reasons. The independence prediction made by the computer simulation described by Kosslyn et al. (in press) must be reconciled with the finding reported by Kosslyn (1987) that strongly right-handed subjects show larger laterality effects for both categorization and distance-judgment tasks than do ambidextrous subjects - a finding that he used to suggest the original nonindependence prediction. Given that an important component in Kosslyn's theory of seeding is the direction and magnitude of hemispheric superiority for verbal processing, it would be worthwhile for future studies to include measures of verbal lateralization for the same subjects who perform the spatial-processing tasks.

Although there is more variability in cerebral laterality (including laterality for verbal processes) among righthanders than was once suspected (e.g., Hellige, Bloch, \& Taylor, 1988; Levine et al., 1987; Levy et al., 1983a, $1983 \mathrm{~b}$ ), the inclusion of left-handed subjects would increase the variation significantly. For this reason, it would also be useful to include left-handers in subsequent studies of individual differences.

It is also important to consider the fact that even studies using only right-handed subjects have reported significant positive correlations between laterality measures for verbal and nonverbal tachistoscopic tasks, even when the tasks produce laterality effects in the opposite direction (e.g., Levine et al., 1987). Positive correlations have also been reported between laterality on verbal and nonverbal tachistoscopic tasks and a free-vision face task thought to measure individual differences in the asymmetric arousal of the two hemispheres (e.g., Hellige, Bloch, \& Taylor, 1988; Levine et al., 1987; Levy et al., 1983a, 1983b). So far, such positive correlations have not emerged as predictions from simulations of Kosslyn's (1987) theory, and their existence suggests that additional factors will need to be taken into account. For example, it has been suggested that such relationships occur because individuals differ in the direction and magnitude of an arousal asymmetry in favor of one hemisphere or the other (Hellige, Bloch, \& Taylor, 1988; Levy et al., 1983a, 1983b) or 
because of individual differences in the relative efficiency of the visual pathways that project to the left or right hemisphere (e.g., Hellige, Bloch, \& Taylor, 1988).

The final purpose of the present experiment was to examine whether or not the mode of processing favored by one hemisphere tended to dominate on bilateral trials when the same information was presented simultaneously to both hemispheres. To do this, we employed the logic developed by Hellige (1987) and utilized in several recent experiments (e.g., Hellige, Jonsson, \& Michimata, 1988; Hellige \& Michimata, 1989; Hellige et al., 1989). The logic grew out of an interest in the ways in which the two hemispheres might interact when both have access to the same stimulus input. One way to study this is to use tachistoscopic presentation tasks for which processing differs qualitatively as a function of which visual field (hemisphere) receives the stimulus input. A useful operational definition of a qualitative difference in processing on unilateral trials is that there is an interaction between visual field and some manipulated task variable. By including trials on which the same stimulus information is presented simultaneously to both visual fields and hemispheres, it is possible to compare the qualitative pattern of task effects when both hemispheres are stimulated (bilateral trials) with the qualitative pattern of task effects on LVF-RH and RVF-LH trials. When the pattern of task effects on bilateral trials is identical to the pattern obtained for one visual field (e.g., RVF-LH trials) but not the other, it suggests that the hemisphere contralateral to that visual field (in this example, left hemisphere) exerts what Levy and Trevarthen (1976) refer to as "metacontrol" for the task.

Recent studies using this logic have found that, for other nonverbal tasks, the pattern of results on bilateral trials was identical to the pattern of results on RVF-LH trials and different from the pattern of results on LVF-RH trials. This was true in an experiment that examined RT to determine that two drawings of faces were different when they were either identical or differed on only one feature (Hellige, Jonsson, \& Michimata, 1988) and also in a physical-identity letter-comparison experiment (Hellige \& Michimata, 1989). One interpretation of these results is that the left hemisphere exerted metacontrol over the qualitative nature of information processing when both hemispheres received the relevant stimuli.

In considering whether the same type of metacontrol might be operating for the present spatial tasks, it is useful to reconceptualize the task $\times$ visual field interaction found on unilateral trials. As Figure 2 indicates, RT was greater for the near/far task than for the above/below task, with this difference being significantly greater on RVF-LH trials ( $M=105 \mathrm{msec})$ than on LVF-RH trials $(M=83 \mathrm{msec})$. In view of findings already discussed, a reasonable interpretation is that the left-hemisphere bias toward use of a categorization-processing subsystem leads to relatively fast processing during the above/below task but relatively slow processing during the near/far task. In contrast, the right-hemisphere bias toward use of a distance-processing subsystem leads to relatively slow processing during the above/below task (compared with left-hemisphere trials), thereby reducing the near/farminus-above/below RT difference. In view of this, it is interesting that the near/far-minus-above/below RT difference on bilateral trials averaged $105 \mathrm{msec}$, identical to the difference on RVF-LH trials and significantly greater than the difference of LVF-RH trials $[F(1,45)=7.48$, $\left.M S_{\mathrm{e}}=654.3, p<.01\right]$. This pattern of effects suggests that, on bilateral trials, subjects are biased toward use of the same categorization-processing subsystems that characterize processing on RVF-LH unilateral trials, with performance being uniformly better when two redundant stimuli are available for processing. The identity of the pattern of task effects on RVF-LH and bilateral trials is reminiscent of similar effects reported in earlier studies with nonverbal stimuli. The consistency of such effects across different experimental paradigms raises the possibility that right-handed adults have a general bias toward modes of processing characteristic of the left hemisphere, at least for nonverbal tasks for which both hemispheres have some competence (see Hellige et al., 1989, for further discussion and for an illustration of how the pattern of interhemispheric interaction can change when verbal stimuli are used).

\section{CONCLUDING COMMENTS}

The results of the present experiment are clearly consistent with the hypothesis that the brain computes two different kinds of spatial-relation representations: one used to assign a spatial relation to a category and the other used to specify metric distances with precision. Furthermore, the specific form of the task $\times$ visual field interaction is consistent with the hypothesis that the left and right cerebral hemispheres make more effective use of the categorization and distance representations, respectively. Accordingly, the computational theory proposed by Kosslyn and his colleagues merits additional attention.

Although it is impressive to find results that conform so nicely to a priori predictions, it must be noted that several issues remain to be investigated. The task $x$ visual field interaction shown in Figure 2 and especially the reversed association shown in Figure 3 provide strong support for the conclusion that the above/below and near/far tasks do not involve identical processing subsystems. This conclusion would be justified even if some other variable were substituted for visual field or if visualfield differences occurred for reasons other than hemispheric asymmetry. However, we must be more cautious about concluding that the specific pattern of results provides unequivocal support for additional hypotheses about hemispheric differences in the use of different types of spatial representation. It is instructive to consider some important questions that remain and the direction that further research might take to address them.

The present visual half-field tasks were designed to maximize the contribution of hemispheric differences and 
minimize the contribution of other variables (see Sergent $\&$ Hellige, 1986). Consequently, we have every reason to believe that the visual-field differences obtained in the present study reflect information-processing differences in the two cerebral hemispheres. However, it is important to seek converging evidence for the conclusion that this is the case. One way to do this would be to examine the performance of patients with unilateral brain damage on tasks that involve categorization versus distance judgment.

The above/below and near/far tasks meet Kosslyn's (1987) criteria for utilizing categorization and distance representations, respectively. However, there is no guarantee that hemispheric differences are restricted to the type of spatial representation that is accessed. It is possible that the two hemispheres both access a single type of spatial representation (contrary to Kosslyn's hypothesis) and that the different hemispheric asymmetries for the two tasks arise in some other task-relevant subsystem. For example, the hemispheres may encode the stimuli in different ways (perhaps emphasizing high vs. low visual spatial frequencies) that are either more or less effective, depending on the decision to be made. Thus, further research is necessary to pinpoint exactly which subsystems are responsible for the hemispheric differences reported here and by Kosslyn (1987).

The final point we will consider is the relationship of the computational approach taken by Kosslyn (1987) and in the present article to more traditional dichotomies that have been suggested to describe hemispheric asymmetries. During the last 30 years, the left hemisphere has been described at different times to be specialized for such things as verbal, analytic, and serial processing, whereas the right hemisphere has been described as being specialized for nonverbal, holistic, and parallel processing. It has become apparent that none of these dichotomies capture anything close to the entire range of hemispheric asymmetries, and it is unlikely that any new dichotomy will. One reason is that even simple tasks involve a number of different processing subsystems, and there is no reason to expect that the hemisphere that is superior for one of the subsystems will be superior for all of them. In fact, there is growing evidence to the contrary (e.g., Allen, 1983; Hellige, 1980). As a result, it is unreasonable to expect neurological correlates of "language" or "spatial processing." Instead, it will prove more fruitful to look for the neurological correlates of the processing subsystems suggested by contemporary computational approaches to human cognition.

\section{REFERENCES}

Allen, M. (1983). Models of hemispheric specialization. Psychological Bulletin, 93, 73-104.
DUNN, J. C., \& KIRSNER, K. (1988). Discovering functionally independent mental processes: The principle of reversed association. Psychological Review, 95, 91-101.

Hellige, J. B. (1980). Effects of perceptual quality and visual field of probe stimulus presentation on memory search for letters. Journal of Experimental Psychology: Human Perception \& Performance, 6 , 639-651.

Hellige, J. B. (1983). Hemisphere by task interaction and the study of laterality. In J. B. Hellige (Ed.), Cerebral hemisphere asymmetry: Method, theory and application (pp. 411-443). New York: Praeger.

HeLlige, J. B. (1987). Interhemispheric interaction: Models, paradigms and recent findings. In D. Ottoson (Ed.), Duality and unity of the brain (pp. 454-465). Hampshire, England: Macmillan.

Hellige, J. B., Bloch, M. I., \& TAYlor, A. K. (1988). Multi-task investigation of individual differences in hemispheric asymmetry. Journal of Experimental Psychology: Human Perception \& Performance, 14, 176-187.

Hellige, J. B., Jonsson, J. E., \& Michimata, C. (1988). Processing from LVF, RVF and BILATERAL Presentations: Metacontrol and interhemispheric interaction. Brain \& Cognition, 7, 39-53.

Hellige, J. B., \& Michimata, C. (1989). Visual laterality for letter comparison: Effects of stimulus factors, response factors, and metacontrol. Bulletin of the Psychonomic Society, 27, 441-444.

Heluige, J. B., \& SERGent, J. (1986). Role of task factors in visual field asymmetries. Brain \& Cognition, 5, 200-222.

Hellige, J. B., TAylor, A. K., \& ENG, T. L. (in press). Interhemispheric interaction when both hemispheres have access to the same information. Journal of Experimental Psychology: Human Perception \& Performance.

Kosslyn, S. M. (1987). Seeing and imagining in the cerebral hemispheres: A computational approach. Psychological Review, 94, 148-175.

Kosslyn, S. M., Sokolov, M. A., \& Chen, J. C. (in press). The lateralization of BRIAN: A computational theory and model of visual hemispheric specialization. In D. Klahr \& K. Katovsky (Eds.), Complex information processing comes of age. Hillsdale, NJ: Erlbaum.

LEVINE, S. C., BANICH, M. T., \& KIM, H. (1987). Variations in arousal asymmetry: Implications for face processing. In D. Ottoson (Ed.), Duality and unity of the brain (pp. 207-222). Hampshire, England: Macmillan.

Levy, J., Heller, W., Banich, M. T., \& Burton, L. (1983a). Are variations among right-handers in perceptual asymmetries caused by characteristic arousal differences between hemispheres? Journal of Experimental Psychology: Human Perception \& Performance, 9, 329-359.

Levy, J., Heller, W., Banich, M. T., \& Burton, L. (1983b). Asymmetry of perception in free viewing of chimeric faces. Brain \& Cognition, 2, 404-419.

LEVY, J., \& TREVARTHEN, C. (1976). Metacontrol of hemispheric function in human split-brain patients. Journal of Experimental Psychology: Human Perception \& Performance, 2, 299-312.

OLDFIELD, R. C. (1971). The assessment and analysis of handedness: The Edinburgh Inventory. Neuropsychologia, 9, 97-113.

Sergent, J., \& Hellige, J. B. (1986). Role of input factors in visual field asymmetries. Brain \& Cognition, 5, 174-199.

ZAIDEL, E. (1983). Disconnection syndrome as a model for laterality effects in the normal brain. In J. B. Hellige (Ed.), Cerebral hemisphere asymmetry: Method, theory and application (pp. 95-151). New York: Praeger. 